\section{Aktuelle Entwicklungen in der Suchtrehabilitation}

Die Suchtrehabilitation hat sich in den letzten Jahrzehnten als Teilsystem der medizinischen Rehabilitation etablieren können. Entwickelt hat sich ein für Deutschland spezifisches, überwiegend von der Rentenversicherung finanziertes Versorgungssystem, das vor allem stationäre Entwöhnungsbehandlungen durchführt. Da Suchterkrankungen häufig mit einer gravierenden Gefährdung der Erwerbsfähigkeit verbunden sind, sah sich die Rentenversicherung gefordert, einer solchen Entwicklung mit Maßnahmen der medizinischen Rehabilitation entgegenzuwirken. Heute gibt es für den stationär-rehabilitativen Indikationsbereich „Abhängigkeitserkrankungen“ knapp 300 Facheinrichtungen mit ca. 14000 Behandlungsplätzen, die jährlich ca. 30000 Rehabilitationsmaßnahmen durchführen.

Die Suchtrehabilitation hatte anfangs innerhalb des Gesamtsystems der medizinischen Rehabilitation um ihre Akzeptanz zu kämpfen. Vielleicht war es gerade diese Außenseiterrolle, die dazu führte, dass hier eher als in anderen Bereichen der medizinischen Rehabilitation schon früh Dokumentationssysteme eingeführt und Evaluationsstudien durchgeführt wurden. $\mathrm{Zu}$ nennen sind in diesem Zusammenhang die Implementierung von umfassenden und suchtspezifischen Dokumentationssystemen wie DOSY, EBIS und heute SEDOS sowie aufwendige Evaluationsstudien wie z.B. die im Auftrag des Verbandes Deutscher Rentenversicherungsträger am Max-Planck-Institut für Psychiatrie ab Mitte der 80er Jahre durchgeführte Münchner Multizentrische Evaluation der Alkoholismus-Therapie (MEAT). Auch im Rahmen des von der Rentenversicherung 1994 begonnenen Qualitätssicherungsprogramms in der Rehabilitation hat der Indikationsbereich Abhängigkeitserkrankungen über seine Fachverbände eine aktive und z.T. die Eigenständigkeit betonende Rolle übernommen und konnte dabei auf die zuvor genannten Vorerfahrungen verweisen.

Dies erkennbare Bemühen um Qualitätssicherung sowie positive Ergebnisse von Evaluationsstudien haben dazu beigetragen, das Stereotyp der vermeintlichen Aussichtslosigkeit suchttherapeutischer/suchtrehabilitativer Ansätze schrittweise abzubauen. Die Einschätzung, die Suchtrehabilitation zähle heute $\mathrm{zu}$ den erfolgreichsten Feldern der medizinischen Rehabilitation, besitzt durchaus Plausibilität. Indirekt kann die Tatsache, dass in der Folge des Wachstums- und Beschäf-

Suchttherapie 2000; 1: 107-109

(c) Georg Thieme Verlag Stuttgart · New York

ISSN 1439-9903
Uwe Koch

tigungsförderungsgesetzes WFG vom September 1996 die Einschnitte im Indikationsbereich der Rehabilitation der Abhängigkeitserkrankungen weniger gravierend waren als in den meisten anderen rehabilitativen Indikationsbereichen, als eine Bestätigung dieser These gewertet werden.

Die medizinische Rehabilitation in Deutschland wie auch die Suchtrehabilitation weisen eine Reihe von Stärken auf. Zu nennen sind hier u.a. das flächendeckend ausgebaute und differenzierte Netzwerk von spezialisierten Einrichtungen, das in ihnen vertretene ganzheitliche Rehabilitationsverständnis, die Verfügbarkeit eines qualifizierten Fachpersonals, das interdisziplinär die diagnostischen und therapeutischen Leistungen erbringt. In der öffentlichen Diskussion wird die medizinische Rehabilitation seit Jahren eher kritisch diskutiert. Diese Argumente beziehen sich u.a. auf den hohen Institutionalisierungsgrad und die starke stationäre Orientierung der medizinischen Rehabilitation und auf Schnittstellenprobleme zwischen Akutversorgung, Rehabilitation und Nachsorge. In den letzten 10 Jahren gab es in der Folge einer Bestandsaufnahme durch eine von der Rentenversicherung eingesetzten Kommission zur Reform der Rehabilitation (VDR, 1991) zahlreiche Initiativen zur Weiterentwicklung der medizinischen Rehabilitation, partiell betreffen diese auch die Suchtrehabilitation.

Ein wichtiges Innovationsziel richtet sich auf eine stärkere Bedarfsorientierung rehabilitativer Maßnahmen. Dahinter steht die Frage, wie diejenigen Versicherten erreicht werden können, bei denen im besonderen Maße eine Rehabilitationsbedürftigkeit gegeben ist. Anders als bei einer Reihe von Rehabilitationsindikationen, bei denen die Frage im Vordergrund steht, ob eine beabsichtigte Maßnahme wirklich erforderlich ist, dürfte die Notwendigkeit einer Maßnahme bei Abhängigkeitserkrankten in der Regel außer Frage stehen. Allenfalls könnte gefragt werden, ob die Maßnahme in jedem Falle in diesem Umfang, in dieser Intensität und stationär erfolgen muss. Besonders relevant ist bei den Abhängigkeitserkrankungen aber das Problem der Nicht-Inanspruchnahme rehabedürftiger Versicherter bzw. des Nichtantritts einer gewährten Maßnahme.

Verbesserungen der Nutzungsadäquanz des rehabilitativen Angebots können auf verschiedenen Wegen erreicht werden, so durch Optimierung der rehabilitationsdiagnostischen Methoden, durch eine bessere administrative Implementierung dieser Diagnostik und durch eine verbesserte Nutzung von Informationen über die Voraussetzungen und das Leistungsangebot der Einrichtungen. Zum zuletzt genannten Aspekt ist 
festzustellen, dass die durch das Qualitätssicherungsprogramm der Rentenversicherung deutlich verbesserte Informationsbasis über die Bedingungen und über das Angebotsspektrum in den einzelnen Einrichtungen der Suchtrehabilitation günstigere Voraussetzungen als bisher für eine Optimierung der Zuweisungssteuerung schafft.

In der Rehabilitationsforschung wird gegenwärtig intensiv an Verfahren gearbeitet, die darauf zielen, den Rehabilitationsbedarf reliabel und valide abzuschätzen. Die hier entwickelten Verfahren sind allerdings wenig auf die spezifischen Belange der Suchtrehabilitation ausgerichtet und deshalb vermutlich nur von begrenztem Vorteil für diesen Versorgungsbereich.

Zu erwähnen sind in diesem Zusammenhang mit der Frage der Bedarfsorientierung aber auch Überlegungen einiger Leistungsträger, Ergebnisse der Suchtforschung zum Themenbereich Prädiktion des Therapieerfolgs bei ihren Entscheidungen über die Gewährung von Entwöhnungsmaßnahmen stärker einfließen zu lassen. Dies kann einerseits zu einer angemesseneren Nutzung der eingesetzten Ressourcen beitragen, andererseits ist eine solche Strategie mit dem Risiko verbunden, dass die therapeutischen Angebote dann im Wesentlichen leichter therapierbaren Patienten gewährt werden. Suchtkranke mit besonderen Risikofaktoren und sozialen Problemlagen würden dann u.U. die für sie besonders notwendige Therapiechance nicht mehr erhalten.

Ein anderes Optimierungsziel, das auch für die Rehabilitation Abhängigkeitserkrankter von großer Bedeutung ist, liegt in der Einleitung möglichst früher Rehabilitationsmaßnahmen. Dahinter steht die Überzeugung, dass das rehabilitative Ziel der sozialen und beruflichen Reintegration durch rehabilitative Maßnahmen umso eher erreicht werden kann, je weniger ausgeprägt und chronifiziert die Krankheitsfolgen sind. Betrachtet man die Anamnese der in den Einrichtungen behandelten Suchtkranken, so findet man vorwiegend lange Krankheitskarrieren, oft verbunden mit inadäquater oder unterlassener suchttherapeutischer Behandlung. Ein möglicher Ansatzpunkt zur Verbesserung liegt in einer stärkeren Einbeziehung von Hausärzten in den Zuweisungsprozess. Diese haben aufgrund ihrer Rolle als primärer Ansprechpartner in Gesundheitsfragen früher als andere professionelle Gruppen die Chance, die Diagnose einer Suchtgefährdung zu stellen, auch könnten sie durch niedrig schwellige Interventionen früh Hilfe leisten oder aufwendigere stationäre Maßnahmen anregen. Voraussetzung dafür ist allerdings eine Verbesserung der suchttherapeutischen Kompetenz der Primärärzte. Erste in diese Richtung zielende Ansätze werden sowohl in der Allgemeinmedizin als auch in der Suchtrehabilitation diskutiert.

Ein anderer Zugangsweg des Suchtkranken in die Rehabilitation ist der aus der stationären Akutversorgung. Auch hier gibt es Optimierungsbedarf. Ärztlicherseits werden zu selten suchttherapeutische Maßnahmen in die therapeutische Planung einbezogen bzw. Patienten aktiv daraufhin angesprochen. Auf der anderen Seite berichten suchtrehabilitative Einrichtungen über die Zuweisung von Patienten, die auf der körperlichen Ebene unzureichend diagnostiziert und therapiert worden sind und damit für die Durchführung rehabilitativer Maßnahmen nicht oder nur eingeschränkt geeignet sind.
Weitere Innovationen in der medizinischen Rehabilitation zielen auf eine Flexibilisierung der weitgehend standardisierten rehabilitativen Angebote in den einzelnen Indikationsbereichen. Die Diskussion um die Flexibilisierung wird inzwischen auch in der Suchtrehabilitation intensiv geführt.

Im Gesamtbereich der Rehabilitation wird in den letzten Jahren intensiv die Frage des Ersetzens oder Ergänzens stationärer Maßnahmen durch teilstationäre und ambulante Rehabilitationsmaßnahmen diskutiert. Davon werden für Teilgruppen von Patienten nicht nur geringere Kosten, sondern auch therapeutische Vorteile, die sich aus der größeren Wohnortnähe nicht stationärer Angebote ergeben, erwartet. In der Rehabilitation Suchtkranker gibt es im Gegensatz zu den meisten anderen rehabilitativen Indikationen eine längere Tradition nicht stationärer Rehabilitationsmaßnahmen. Nachdem die deutsche Hauptstelle gegen Suchtgefahren DHS bereits 1979 Mindestkriterien für die ambulante Suchtberatung und Behandlungsstellen formuliert hatte, wurde auf Ebene der Kosten- und Leistungsträger 1991 die Empfehlungsvereinbarung „Ambulante Rehabilitation Sucht“ verabschiedet. Sie regelt Fragen der Zuständigkeit, Voraussetzungen, Art, Umfang, Verfahren und Finanzierung ambulanter Leistungen im Rahmen der Rehabilitation Abhängigkeitserkrankter. Gegenwärtig gibt es bundesweit ein dichtes Netzwerk von über 1200 anerkannten ambulanten Einrichtungen. Der Anteil ambulant durchgeführter Maßnahmen steigt seit Jahren kontinuierlich und hat nach Angaben der Rehabilitationsstatistik des Verbands Deutscher Rentenversicherungsträger für das Jahr 1998 einen Anteil von ca. 13\% erreicht. Die bei der Einführung formulierten optimistischen Erwartungen bez. der Entwicklung der ambulanten Suchtrehabilitation haben sich damit nur z.T. erfüllt. Als Gründe werden von Suchtexperten v.a. die relativ hohen Eingangsvoraussetzungen gesehen. Evaluationsstudien zum langfristigen Erfolg ambulant durchgeführter Behandlungen stehen noch aus. In jüngerer Zeit wurden ergänzend zu den in der Empfehlungsvereinbarung von 1991 beschriebenen Maßnahmen auch tagesklinische/teilstationäre Modelle der Suchtrehabilitation entwickelt.

Andere aktuelle Veränderungsbemühungen zielen auf die weitere Beseitigung von Schnittstellen und damit auf eine bessere Integration der bisherigen Suchtrehabilitation in das Versorgungssystem. Zu nennen sind hier v.a. Maßnahmen, die den Patienten gezielt auf die Entwöhnungsbehandlung vorbereiten. Dazu gehört insbesondere die Stärkung der Motivation im Rahmen des qualifizierten Entzugs. Bezüglich der Übernahme der dabei entstehenden zusätzlichen Kosten wurden an einigen Orten Auseinandersetzungen zwischen Krankenkassen und Rentenversicherung berichtet. In der seit längerem bestehenden Arbeitsgruppe Sucht der Spitzenverbände Kranken- und Rentenversicherung ist dieses Problem aber inzwischen einer Lösung zugeführt worden.

Schnittstellenfragen stellen sich auch im Zusammenhang mit der Nachsorge. Viele Einrichtungen bemühen sich hier sehr intensiv um enge Kooperation mit Selbsthilfegruppen. Im Bereich der illegalen Drogen werden inzwischen in fast allen Einrichtungen durch die Adaptationsbehandlung verbesserte Möglichkeiten für die Reintegration der Betroffenen geschaffen. Zur Güte der Zusammenarbeit im Rahmen der Nachsorge von Einrichtungen der stationären Suchtrehabilitation mit 
Suchtberatungsstellen, niedergelassenen Ärzten und dem werkärztlichen Dienst liegen nur begrenzt Informationen vor.

Das hier vorgelegte Schwerpunktheft behandelt mit sechs Beiträgen ausgewählte Themen der Rehabilitation Suchtkranker. Die ersten drei Beiträge befassen sich v.a. mit der Struktur- und Prozessqualität. K. Huck-Langer, B. Maier-Riehle, F. Schliehe stellen Ergebnisse einer vom Verband Deutscher Rentenversicherungsträger vorgenommenen Auswertung zur Struktur der rehabilitativen Einrichtungen im Indikationsbereich Abhängigkeitserkrankungen sowie zu den dort angebotenen therapeutischen Leistungen dar. U. Zemlin und Kollegen untersuchen das therapeutische Angebot auf der Ebene des Patienten, indem sie vor dem Hintergrund des ICIDH-Konzepts die in mehreren Kliniken der Suchtrehabilitation bei Alkohol- und Medikamentenabhängigkeiten erbrachten therapeutischen Leistungen analysieren. Fragen der Prozessqualität sind auch Thema des Beitrages von S. Kawski und Kollegen. Hier wird das im Rahmen des Qualitätssicherungsprogramms der Rentenversicherung eingesetzte Peer-ReviewVerfahren zur Beurteilung der Qualität der Durchführung der Rehabilitationsmaßnahmen in seiner spezifischen Anpassung an die Rehabilitation Suchtkranker vorgestellt und empirisch geprüft.

Mit der als erforderlich angesehenen strukturellen Weiterentwicklung der Suchtrehabilitation befassen sich die nachfolgenden drei Beiträge. T. Hansmeier, W. Müller-Fahrnow, K. Spyra nehmen zunächst eine versorgungsepidemiologische Problemanalyse vor. Einen vordringlichen Forschungs- und Entwicklungsbedarf sehen sie in der bedarfsorientierten Zuweisung, in der Stufung der Suchtrehabilitation und in der Überwindung von Schnittstellen. J. Köhler beschreibt anschließend aus der Perspektive eines großen Rentenversicherungsträgers (BfA) die Rahmenbedingungen für den weiteren Ausbau einer ambulanten Rehabilitation Sucht, die bisherige Entwicklung dieses Bereichs seit 1995 und die Einstellungen Versicherter gegenüber ambulant durchgeführten suchtrehabilitativen Maßnahmen. M. Beutel und M. Baumann zeigen auf, dass die Suchtrehabilitation älterer Menschen im bisherigen Angebotsspektrum nicht hinreichend Berücksichtigung findet, und beschreiben, unter welchen Bedingungen ältere suchtkranke Patienten durch ambulante und stationäre Behandlungsangebote erfolgreich rehabilitiert werden können.
Prof. Dr. Dr. Uwe Koch

Universitätsklinikum Hamburg-Eppendorf

Abteilung für Medizinische Psychologie

Martinistraße 52

20246 Hamburg 